\title{
Diversity-Multiplexing Trade-Off in Coherent Free-Space Optical Systems With Multiple Receivers
}

\author{
Sahar Molla Aghajanzadeh and Murat Uysal
}

\begin{abstract}
In this paper, from an information theory point of view, we investigate the performance of a coherent freespace optical (FSO) communication system with multiple receive apertures over atmospheric turbulence channels. Our study builds on a recently introduced statistical model that characterizes the combined effects of turbulence-induced wavefront distortion and amplitude fluctuation in coherent receivers with phase compensation. We investigate the link reliability as quantified by "diversity gain" and the relationship between the link reliability and the spectral efficiency as quantified by "diversity-multiplexing trade-off (DMT)." Our results provide insight into the performance mechanisms of coherent FSO systems and demonstrate significant performance gains that can be obtained through the deployment of multiple receive apertures and phase compensation techniques.
\end{abstract}

Index Terms-Free-space optical systems; Atmospheric turbulence-induced fading; Receive diversity; Diversity gain; Diversity-multiplexing trade-off.

\section{INTRODUCTION}

$\mathbf{F}$ ree-space optical (FSO) communication [1] refers to optical transmission over the atmosphere through the use of either lasers or light-emitting diodes (LEDs). As a licensefree, cost-effective, and easy-to-install alternative to fiber optics, FSO systems have attracted attention as an efficient solution for a wide range of applications [2]. Based on the type of detection, FSO systems can be categorized as either noncoherent (direct detection) or coherent (heterodyne detection) systems. In heterodyne systems, the receiver mixes the incoming optical field with a local oscillator (LO) field and then photodetects the combined wave. In comparison to intensity modulation/direct detection (IM/DD) systems, heterodyne receivers are more difficult to implement since the LO field should be spatially and temporally coherent with the received field. However, they provide more flexibility as any kind of amplitude, frequency, or phase modulation can be used in these systems. Furthermore, they can provide significant performance enhancements due to spatial temporal selectivity and heterodyne gain [3].

Manuscript received May 25, 2010; revised August 27, 2010; accepted October 8, 2010; published November 30, 2010 (Doc. ID 128896).

S. M. Aghajanzadeh (e-mail: smollaag@uwaterloo.ca) is with the Department of Electrical and Computer Engineering, University of Waterloo, Waterloo, Ontario, N2L 3G1, Canada.

M. Uysal (e-mail: murat.uysal@ozyegin.edu.tr) is with the Faculty of Engineering, Özyeğin University, 34662, Istanbul, Turkey.

Digital Object Identifier 10.1364/JOCN.2.001087
A major performance impairment in FSO links is atmospheric turbulence-induced fading. A typical fade can last milliseconds, and, considering that FSO links operate at the speed of several gigabits/second, a single fade can result in the loss of a large number of consecutive bits. Spatial diversity techniques are commonly used to mitigate the degrading effects of fading. This type of diversity involves the use of multiple transmit and/or receive apertures and has been investigated in detail for IM/DD systems; see, e.g., [4-13] and the references therein. However, the current literature on coherent FSO systems with spatial diversity is sparse [14-17].

Related Literature and Contributions: In [14,15], Lee and Chan have studied receive diversity in coherent FSO systems through the derivation of outage probability. Haas et al. have addressed the concept of transmit diversity in [16] and proposed space-time channel codes (inspired from radiofrequency wireless communication literature) through the minimization of pairwise error probability. Both works consider log-normal distribution for amplitude fading and uniform distribution for the phase. An underlying assumption in [14-16] is that the field of the received signal is spatially coherent with that of the LO. In practice, atmospheric turbulence distorts the coherency of the received wavefront and the resulting imperfect wavefront match between the incoming signal and the LO degrades the coherent system performance. Particularly in cases when the diameter of the receive aperture is larger than the coherence length of the received wavefront, phase distortion becomes a performance-limiting factor and phase compensation needs to be deployed.

In [18], Belmonte and Kahn have developed a statistical model to characterize the combined effects of turbulenceinduced phase distortion and amplitude fluctuation on the performance of coherent receivers with partial phase compensation. Using this channel model, they have further investigated the outage probability and the spectral efficiency of coherent FSO systems with multiple receive apertures in [17]. In this paper, we adopt the channel model of [18] and study the performance of coherent FSO systems with multiple receive apertures from an information theoretic point of view. Specifically, we investigate the link reliability, as quantified by the "diversity gain" [19], and the relationship between the link reliability and spectral efficiency, as quantified by the "diversity-multiplexing trade-off (DMT)" [20]. We derive a DMT expression for the finite signal-to-noise ra- 
tio (SNR) regime that provides insight into the performance mechanisms of coherent FSO systems under practical operation range. The derived expression is shown to be a function of the number of receive apertures $(M)$ as well as the effective channel parameter $(K)$, which depends on the turbulence-induced fading characteristics and the number of compensated modes. For a sufficiently large SNR, we demonstrate that the DMT converges to $M(1-r)$ where $r$ is the multiplexing gain. Therefore, the maximum diversity gain (i.e., DMT evaluated at $r=0$ ) is determined by the number of receive apertures. However, interestingly, in the finite-SNR regime (which is also the practical operation range), the diversity order can exceed $M$. This is a result of the fact that it is determined by not only $M$ but also $K$, which can be increased by increasing the number of compensated modes. Our results demonstrate that modal compensation acts as an additional diversity source besides multiple apertures at the receiver side.

The rest of the paper is organized as follows: In Section II, we describe the coherent FSO system model under consideration and the Rician channel model adopted in the analysis. In Section III, we derive closed-form expressions for outage probability, finite-SNR DMT, and finite-SNR diversity gain. In Section IV, we present numerical results and finally conclude in Section V.

\section{SYSTEM DESCRIPTION}

\section{A. Signal Model}

Figure 1 illustrates the block diagram of a coherent FSO system with $M$ heterodyne receivers. Receive apertures are separated by more than a coherence length to ensure the independency of fading channels. The received field at the aperture plane of the $k$ th $(1 \leqslant k \leqslant M)$ receiver is given by [1]

$$
E_{r_{k}}(t, \mathbf{r})=u_{s}(t) e^{j\left(2 \pi f_{c} t+\theta_{s}(t)\right)} e^{\left[\chi_{k}(\mathbf{r})+j \varphi_{k}(\mathbf{r})\right]},
$$

where $\mathbf{r}$ is the position vector on the receive aperture plane, $f_{c}$ is the optical carrier frequency, and $u_{s}(t) e^{j \theta_{s}(t)}$ is the com-

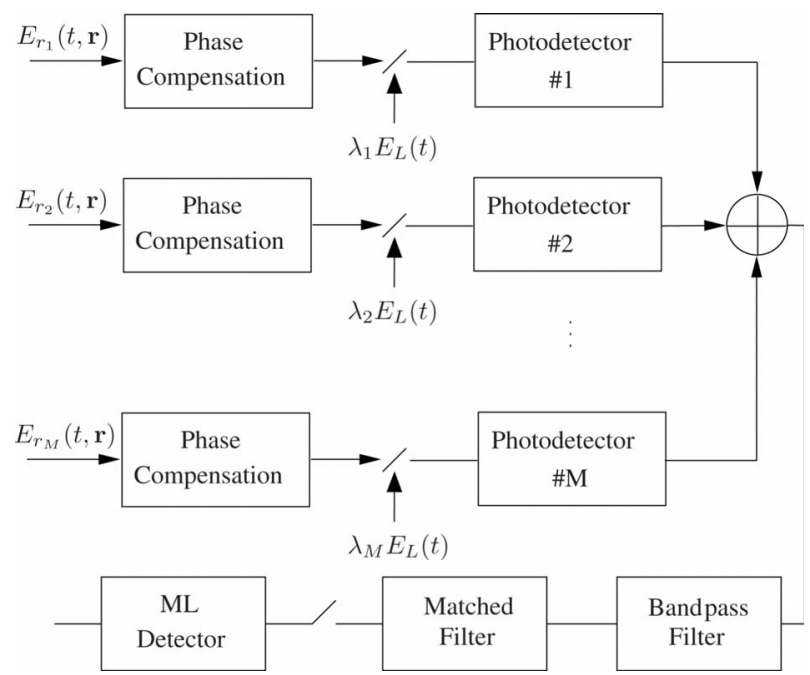

Fig. 1. Block diagram of a coherent FSO system with multiple heterodyne receivers. plex envelope of the modulation signal [chosen from the phase-shift-keying (PSK) or quadrature amplitude modulation (QAM) constellation]. Here, $\chi_{k}(\mathbf{r})$ and $\varphi_{k}(\mathbf{r})$ represent the turbulence-induced log-normal amplitude fluctuations and Gaussian phase variations of the $k$ th channel, respectively.

In an optical heterodyne receiver, the field of the received signal should be spatially and temporally coherent with that of the LO in order to maximize the received power [1]. Atmospheric turbulence distorts the coherency of the received signal that is to be mixed with the LO field. To compensate the phase distortion due to the turbulence, either zonal or modal compensation can be deployed [21]. In this paper, we assume modal compensation in which the total phase distortion is expanded into a set of basis functions (modes), such as Zernike polynomials [22], and then some modes of this expansion are corrected. It has been shown that the residual phase variance after the compensation of $J$ Zernike terms over an aperture with diameter $D$ is given by [22]

$$
\sigma_{\varphi}^{2}=\Delta_{J}\left(D / r_{0}\right)^{2}
$$

where the coefficient $\Delta_{J}$ is determined by the number of compensated modes, and $r_{0}$ is the Fried parameter that corresponds to the wavefront coherence diameter. For plane waves and Kolmogrov turbulence, $r_{0}$ can be expressed in terms of the wavenumber $(k)$, the refractive index structure constant $\left(C_{n}^{2}\right)$, and the propagation distance $(Z)$ as $r_{0}$ $=1.68\left(C_{n}^{2} Z k^{2}\right)^{-3 / 5}[23]$.

Let $E_{L}(t)=A_{L} e^{j\left(2 \pi f_{L} t+\theta_{L}\right)}$ be the LO field in the single receiver scenario with amplitude $A_{L}$, frequency $f_{L}$, and phase $\theta_{L}$. The LO field of the $k$ th receiver is multiplied by a proper weighting factor $\lambda_{k}, 1 \leqslant k \leqslant M$, i.e., $E_{L_{k}}(t)=\lambda_{k} E_{L}(t)$ under the constraint of $\sum_{k=1}^{M}\left|\lambda_{k}\right|^{2}=1$. This constraint ensures that the total LO power in the multiple receiver scenario is equivalent to the LO power in the single receiver case [14].

The summation of the received optical field and the LO field in each diversity branch passes into a photodetector. The total output current of the system is the summation of the output currents of $M$ photodetectors. After the combination of diversity branches' outputs, a bandpass filter is employed to extract the intermediate frequency (IF) component of the total output current. The IF component of the $k$ th photodetector's output is given by

$$
y_{k}(t)=x_{k}(t)+n_{k}(t)
$$

where $x_{k}(t)$ is the information-carrying part and $n_{k}(t)$ is the noise term dominated by the LO shot noise, which can be modeled as additive white Gaussian noise (AWGN) [1]. Consequently, the total IF output signal is given by

$$
y_{T}(t)=\sum_{k=1}^{M} y_{k}(t)=\underbrace{\sum_{k=1}^{M} x_{k}(t)}_{x_{T}(t)}+\underbrace{\sum_{k=1}^{M} n_{k}(t)}_{n_{T}(t)},
$$

where the Gaussian noise term $n_{T}(t)$ has zero mean and variance of [1] 


$$
P_{n_{T}}=\frac{B_{s} e^{2} \eta \pi}{2 h f_{c}} D_{k}^{2} A_{L}^{2}
$$

Here, $e$ is the electron's charge, $h$ is Planck's constant, $\eta$ is the quantum efficiency of the photodetector, $B_{s}$ is the signal bandwidth, and $D_{k}$ is the aperture diameter of each receiver. ${ }^{1}$ Using the results from $[1,18], x_{T}(t)$ can be written as

$$
x_{T}(t)=\frac{e \eta \pi}{2 h f_{c}} D_{k}^{2} A_{L} u_{s}(t) \operatorname{Re}\left\{e^{j\left(2 \pi f_{I F} t+\theta_{s}(t)-\theta_{L}\right)} \sum_{k=1}^{M} \alpha_{k} \lambda_{k}^{*}\right\},
$$

where $f_{I F}=f_{c}-f_{L}$ is the intermediate frequency and $\alpha_{k}$ represents the effective fading coefficient modeling the channel of the $k$ th diversity branch. Statistical characterization of $\alpha_{k}$ has been developed in [18], which will be briefly summarized in the following subsection. The output instantaneous SNR of the system after heterodyning and combining diversity branches is given by

$$
\gamma=\frac{P_{x_{T}}}{P_{n_{T}}}=\frac{\eta I_{s}}{B_{s} h f_{c}}\left(\frac{\pi D^{2}}{4}\right)\left|\sum_{k=1}^{M} \alpha_{k} \lambda_{k}^{*}\right|^{2}
$$

where $I_{s}=\overline{\left|u_{s}(t)\right|^{2}}$ denotes the average intensity of the optical signal field and $P_{x_{T}}$ is the output signal power. By using the Cauchy-Schwartz inequality, the values of $\lambda_{k}$ s, which maximize SNR can be found as

$$
\lambda_{k, o p t}=\frac{\alpha_{k}}{\sqrt{\sum_{i=1}^{M}\left|\alpha_{i}\right|^{2}}} .
$$

Consequently, the corresponding SNR at the maximal ratio combiner output is given by

$$
\gamma=\frac{\rho}{M} \sum_{k=1}^{M} a_{k}^{2}
$$

where $a_{k}=\left|\alpha_{k}\right|$ and $\rho=\eta I_{s} \pi D^{2} /\left(4 B_{s} h f_{c}\right)$ is the SNR in the absence of turbulence.

\section{B. Channel Model}

Let $\alpha_{r, k}$ and $\alpha_{i, k}$ denote the real and imaginary parts of $\alpha_{k}$, respectively. It is shown in [18] that they follow Gaussian distribution, i.e., $\alpha_{r, k} \sim N\left(\bar{\alpha}_{r}, \sigma_{r}^{2}\right)$ and $\alpha_{i, k} \sim N\left(\bar{\alpha}_{i}, \sigma_{i}^{2}\right)$. Their means and variances are given by $\bar{\alpha}_{r}=e^{-\left(\sigma_{\chi}^{2}+\sigma_{\varphi}^{2}\right) / 2}, \sigma_{r}^{2}$ $=(1 / 2 G)\left(1+e^{-2 \sigma_{\varphi}^{2}}-2 e^{-\sigma_{\chi}^{2}-\sigma_{\varphi}^{2}}\right), \bar{\alpha}_{i}=0$, and $\sigma_{i}^{2}=(1 / 2 G)\left(1-e^{-2 \sigma_{\varphi}^{2}}\right)$. Here

- $\sigma_{\chi}^{2}$ is the log-amplitude variance, which is given by $\sigma_{\chi}^{2}$ $=0.307 k^{7 / 6} Z^{11 / 6} C_{n}^{2}[23]$.

- $\sigma_{\varphi}^{2}=\Delta_{J}\left(D_{k} / r_{0}\right)^{2}$ is the residual phase variance after the compensation of $J$ Zernike terms over each receive aperture with diameter $D_{k}$.

${ }^{1}$ For the sake of fair comparison in terms of received signal power, the aperture area of each receiver in the multiple receiver system is assumed to be $1 / M$ times the aperture area of a single receiver system, i.e., $D_{k}=D / \sqrt{M}, 1 \leqslant k \leqslant M$, where $D$ denotes the receive aperture diameter in the benchmark single receiver system $(M=1)$ $[14,17]$.
- $G$ is the number of statistically independent patches (i.e., the area within which the received wavefront is approximately coherent) existing in each receive aperture plane and is given $\mathrm{by}^{2}$ [18]

$$
G=\left\{1.09\left(\rho_{0} / D_{k}\right)^{2} \Gamma\left[1.2,1.08\left(D_{k} / \rho_{0}\right)^{5 / 3}\right]\right\}^{-1},
$$

where $\Gamma(.,$.$) denotes the lower incomplete Gamma$ function, and $\rho_{0}$ is the generalized Fried parameter that corresponds to partially compensated wavefronts. The generalized Fried parameter after the compensation of $J$ Zernike terms is given by $\rho_{0}$ $\approx\left(3.44 / \Delta_{J}\right)^{3 / 5} 0.286 J^{-0.362} r_{0}[24]$.

Under the assumption that $G$ is large enough and $\sigma_{r}^{2}$ $=\sigma_{i}^{2}, a_{k}=\left|\alpha_{k}\right|$ follows the Rician distribution. However, even for $\sigma_{r}^{2} \neq \sigma_{i}^{2}$, it can be approximated by the Rician distribution for most practical purposes [18]. The corresponding Rician probability density function (pdf) is given by

$$
f_{a_{k}}\left(a_{k}\right)=\frac{2 a_{k}(1+K)}{\overline{a^{2}}} e^{-K-(1+K) a_{k}^{2} / \overline{a^{2}}} I_{0}\left(2 a_{k} \sqrt{\frac{K(1+K)}{\overline{a^{2}}}}\right),
$$

where $I_{0}($.$) is the zeroth-order modified Bessel function of$ the first kind [25] and $\overline{a^{2}}=\sigma_{r}^{2}+\sigma_{i}^{2}+\bar{\alpha}_{r}^{2}$. The parameter $K$ is the ratio of the strength of the coherent component to the incoherent one in the detected field and given by [18]

$$
K=\left[\frac{\overline{a^{2}}}{\sqrt{\bar{\alpha}_{r}^{4}+2 \bar{\alpha}_{r}^{2}\left(\sigma_{i}^{2}-\sigma_{r}^{2}\right)-\left(\sigma_{i}^{2}-\sigma_{r}^{2}\right)^{2}}}-1\right]^{-1} .
$$

\section{DiVERSITY AND MULTIPLEXING GAINS}

In this section, we first provide some basic definitions of diversity and multiplexing gains, then present the related derivations for the coherent FSO system under consideration. Diversity and multiplexing gains are two performance measures commonly used in the performance analysis of wireless radio-frequency $(\mathrm{RF})$ multiple antenna systems [26]. There are two potential gains that can be exploited from a multiple antenna system. If the channels between individual transmit-receive antenna pairs experience independent fading, multiple parallel spatial channels are effectively created. By sending different information through these channels, the data rate can be increased in comparison to a single antenna system. This increase in data rate is known as multiplexing gain. On the other hand, one can take advantage of the multiple antenna system to extract diversity gain. By sending the same information through multiple independent channels, independently faded replicas of the information will be obtained at the receiver. Through proper processing of these multiple replicas, one can decrease the error probability. The resulting performance advantage is known as the diversity gain. In general, a scheme that maximizes one gain over a specific communication channel does not guarantee to maximize the other. In fact, it is shown in [20] that there is a trade-off between these two gains called DMT that prevents maximizing both gains simultaneously. DMT characterizes the maximum possible di-

\footnotetext{
${ }^{2}$ Note that $r_{0}$ in Eq. (22) of [18] should be replaced by $\rho_{0}$.
} 
versity gain that can be achieved at a given multiplexing gain over a communication channel. Although, this concept was originally proposed for multiple-input-multiple-output (MIMO) systems, DMT can be defined for any communication system including single-input-single-output (SISO). Although the multiplexing gain depends on the coding rate across time and/or space in a MIMO system, it is determined by the coding rate only across time in a SISO system [20].

\section{A. Asymptotical Versus Finite-SNR DMT}

Conventional definitions of diversity and multiplexing gains apply to the asymptotical case when the SNR approaches infinity. The diversity gain is defined as [20]

$$
d=-\lim _{\rho \rightarrow \infty} \frac{\log P_{\text {out }}(R, \rho)}{\log \rho},
$$

where $R$ is the target data rate, $\rho$ is the $\operatorname{SNR}$, and $P_{\text {out }}(R, \rho)$ is the outage probability. On the other hand, if the data rate $R$ is not fixed and increases with SNR, the multiplexing gain is defined as [20]

$$
r=\lim _{\rho \rightarrow \infty} \frac{R(\rho)}{\log \rho} .
$$

From Eqs. (13) and (14), one can observe that $d$ is in fact a function of $r$ when the data rate is variable. In such cases, it is said that the diversity gain $d(r)$ is achieved at the multiplexing gain $r$. The curve of $d(r)$ is known as (asymptotical) DMT [20].

Since conventional definitions apply to the asymptotically high SNRs, definitions for diversity and multiplexing gains for finite-SNR regimes have been further introduced that are particularly useful in evaluating these gains at practical SNR values. The finite-SNR diversity gain is given by [27]

$$
d_{f}(R, \rho)=-\frac{\partial \log P_{\text {out }}(R, \rho)}{\partial \log \rho} .
$$

It represents the negative slope of the log-log plot of the outage probability versus SNR at a target data rate $R$. When the data rate increases with SNR, the multiplexing gain in the finite-SNR regime is defined as the ratio of the data rate $R(\rho)$ to the capacity of an AWGN channel at a given SNR. It is given by [27]

$$
r_{f}=\frac{R(\rho)}{\log (1+\rho)} .
$$

By inserting Eq. (16) into Eq. (15), the finite-SNR DMT can be obtained as

$$
d_{f}\left(r_{f}, \rho\right)=-\frac{\rho}{P_{\text {out }}\left(r_{f}, \rho\right)} \frac{\partial P_{\text {out }}\left(r_{f}, \rho\right)}{\partial \rho} .
$$

It can be readily checked that the definitions of finite-SNR diversity and multiplexing gains are consistent with the asymptotical ones, that is, $\lim _{\rho \rightarrow \infty} d_{f}=d$ and $\lim _{\rho \rightarrow \infty} r_{f}=r$.

\section{B. Derivation of Outage Probability}

The outage probability at a given data rate $R$ is defined as [19]

$$
P_{\text {out }}(R)=\operatorname{Pr}\{I(\gamma)<R\},
$$

where $I(\gamma)=\log _{2}(1+\gamma)$ is the mutual information between the source and destination. Since $I(\cdot)$ is monotonically increasing with $\gamma$, Eq. (18) can be expressed as

$$
P_{\text {out }}(R)=\operatorname{Pr}\left\{\gamma<\gamma_{R}\right\},
$$

where $\gamma_{R}=I^{-1}(R)=2^{R}-1$ denotes the threshold SNR that is required to support the rate $R$. Substituting Eq. (9) into Eq. (19), we have

$$
P_{\text {out }}(R)=\operatorname{Pr}\left\{y<M \gamma_{R} / \rho\right\},
$$

where $y=\sum_{k=1}^{M} a_{k}^{2}$ is a complex noncentral chi-square random variable with $M$ degrees of freedom and the noncentrality parameter $K M$. The pdf of $y$ is given by [28]

$$
\begin{aligned}
f_{Y}(y)= & \frac{(1+K)}{\overline{a^{2}}}\left(\frac{(1+K) y}{M K \overline{a^{2}}}\right)^{(M-1) / 2} \\
& \times e^{-K M-(1+K) y / \bar{a}^{2}} I_{M-1}\left(2 \sqrt{\frac{K(1+K) M y}{a^{2}}}\right),
\end{aligned}
$$

where $I_{M-1}(\cdot)$ is the $(M-1)$ th-order modified Bessel function of the first kind [25]. Hence, the outage probability is given by

$$
\begin{aligned}
P_{\text {out }}(R, \rho) & =\int_{0}^{M \gamma_{R} / \rho} f_{y}(y) \mathrm{d} y \\
& =1-Q_{M}\left(\sqrt{2 K M}, \sqrt{\frac{2(1+K) M \gamma_{R}}{\rho \bar{a}^{2}}}\right),
\end{aligned}
$$

where $Q_{M}(\zeta, \beta)$ is the generalized Marcum $\mathrm{Q}$-function of or$\operatorname{der} M$ [29]. It should be noted that our derived expression given by Eq. (22) is different from Eq. (6) of [17], which seems to be erroneous. ${ }^{3}$

\section{Derivation of Finite-SNR DMT}

From Eq. (16), we have $R(\rho)=r_{f} \log (1+\rho)$. The threshold SNR $\gamma_{R}$ can be obtained in terms of $r_{f}$ and $\rho$ as

$$
\gamma_{R}=2^{R}-1=(1+\rho)^{r_{f}}-1 .
$$

Substituting Eq. (23) into Eq. (22) and inserting the resulting expression into Eq. (17), we have

$$
d_{f}\left(r_{f}, \rho\right)=\frac{\rho}{1-Q_{M}(\zeta, \beta)} \frac{\partial Q_{M}(\zeta, \beta)}{\partial \beta} \frac{\partial \beta}{\partial \rho},
$$

where $\zeta=\sqrt{2 K M}$ and $\beta$ is given by

${ }^{3}$ One can readily check that Eq. (6) of [17] is erroneous by inspecting the limiting cases of the parameter $K$ (defined as $r$ in [17]). Note that $r \rightarrow 0$ and $r \rightarrow \infty$ respectively correspond to Rayleigh and Gaussian channels. For $r \rightarrow 0$, the derived outage probability in [17] goes to $-\infty$, whereas it goes to 1 for $r \rightarrow \infty$, which are obviously wrong. The mistake probably comes from the erroneous pdf given by Eq. (5) of [17] used for the noncentral chi-square random variable. 


$$
\beta=\sqrt{\frac{2(1+K) M\left[(1+\rho)^{r} f-1\right]}{\rho \overline{a^{2}}}} .
$$

After differentiation and some mathematical manipulation, Eq. (24) yields

$$
d_{f}\left(r_{f}, \rho\right)=\frac{\beta^{M+1} e^{-\left(\beta^{2}+\zeta^{2}\right) / 2} I_{M-1}(\zeta \beta)}{2 \zeta^{M-1}\left[1-Q_{M}(\zeta, \beta)\right]}\left(1-\frac{r_{f} \rho(1+\rho)^{r_{f}-1}}{(1+\rho)^{r_{f}-1}}\right) .
$$

Next, we investigate the asymptotic value of the DMT at the high-SNR regime. Expressing the Marcum Q-function in terms of its series form [29] and using the equivalent series form of the modified Bessel function [25], Eq. (26) can be rewritten as

$$
\begin{aligned}
d_{f}\left(r_{f}, \rho\right)= & \frac{\sum_{k=0}^{\infty} \frac{1}{k !(M+k-1) !}\left(\frac{\zeta \beta}{2}\right)^{2 k}}{\sum_{j=0}^{\infty}\left(\frac{\beta^{2}}{2}\right)^{j}\left[\sum_{k=0}^{\infty} \frac{1}{k !(j+k+M) !}\left(\frac{\zeta \beta}{2}\right)^{2 k}\right]} \\
& \times\left(1-\frac{r_{f} \rho(1+\rho)^{r_{f}-1}}{(1+\rho)^{r_{f}-1}}\right) .
\end{aligned}
$$

For $\rho \rightarrow \infty$, we have $\beta \rightarrow \sqrt{B \rho^{r-1}}$, where $B=2(1+K) M / \overline{a^{2}}$. Noting $0 \leqslant r<\min \{1, M\}[20]$, it can be found out that $\lim _{\rho \rightarrow \infty} \beta$ $=0$. Therefore, the asymptotic value of DMT at high SNR can be obtained as

$$
\lim _{\rho \rightarrow \infty} d\left(r_{f}, \rho\right)=M(1-r) .
$$

This result agrees with the asymptotic behavior of DMT for multiple receive antenna systems analyzed in the context of RF communications [20].

\section{Derivation of Finite-SNR Diversity Gain for a Fixed Data Rate}

When the data rate $R$ is kept fixed, the threshold SNR value $\gamma_{R}$ is constant (i.e., not a function of $\rho$ ). Substituting Eq. (22) into Eq. (15), we obtain the finite-SNR diversity gain, which turns out to have an identical form of Eq. (24), where $\beta$ is now given by $\beta=\sqrt{2(1+K) M \gamma_{R} /\left(\rho \bar{a}_{k}^{2}\right)}$. Consequently, we obtain

$$
d_{f}(R, \rho)=\frac{\beta^{M+1} e^{-\left(\beta^{2}+\zeta^{2}\right) / 2} I_{M-1}(\zeta \beta)}{2 \zeta^{M-1}\left[1-Q_{M}(\zeta, \beta)\right]} .
$$

In the high-SNR regime (i.e., $\rho \rightarrow \infty$ ), we have

$$
\lim _{\rho \rightarrow \infty} d_{f}(R, \rho)=M
$$

demonstrating that the diversity gain is determined by the number of receive apertures. For a sanity check, note that, in the high-SNR regime, the diversity gain at a fixed rate $R$ is expected to be equal to the DMT evaluated at $r=0$. For $r=0$, Eq. (28) yields $M$, which coincides with Eq. (30).
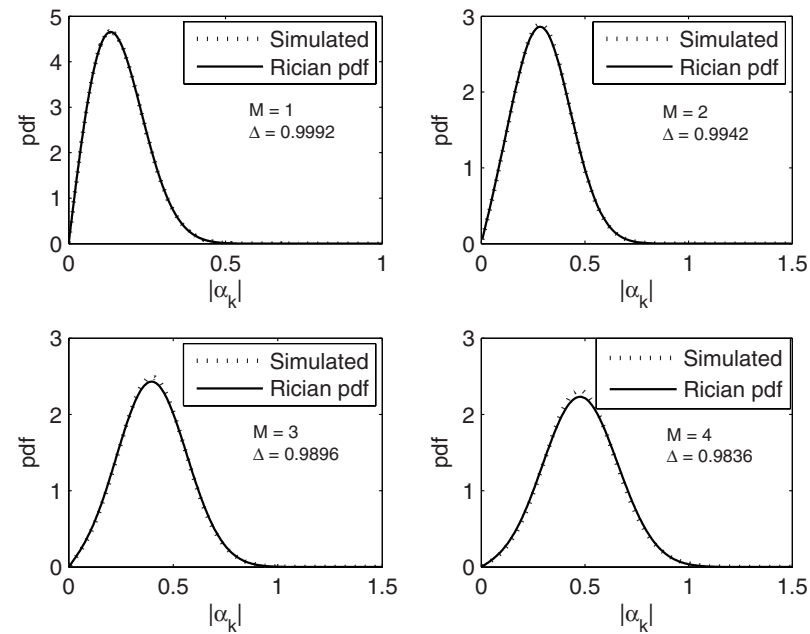

Fig. 2. (Color online) Simulated and Rician pdfs for different numbers of receive apertures.

\section{NUMERICAL RESUlTS AND DISCUSSIONS}

In this section, we present numerical results for the outage probability, finite-SNR DMT, and diversity gain derived in the previous sections. We assume an FSO system with wavelength $\lambda=1.55 \mu \mathrm{m}$ operating under turbulence conditions with $C_{n}^{2}=5 \times 10^{-14} \mathrm{~m}^{-2 / 3}$. The path length is $Z=1 \mathrm{~km}$ and the aperture diameter of "the equivalent" single receiver is chosen as $D=25 \mathrm{~cm}$.

Before we present information theoretic results, we first validate the underlying statistical model for the system with the above characteristics. In Fig. 2, we use the Monte Carlo method to simulate the exact channel of each diversity branch for different numbers of receive apertures $(M)$ assuming that $J=3$ Zernike modes are compensated in each aperture. The Rician pdf used in the theoretical derivation is provided as well. It is observed from Fig. 2 that the simulated data and Rician pdf provide a very good match. To quantify the statistical matching, we have also calculated the overlapping coefficient $\Delta$ [30] between two pdfs. $\Delta=1$ means that two pdfs are exactly the same, and $\Delta=0$ means two pdfs are completely distinct. In our case, we have found $\Delta=0.9992, \Delta=0.9942, \Delta=0.9896$, and $\Delta=0.9836$ for $M=1,2$, 3 , and 4 , respectively.

Figure 3 demonstrates the outage probability given by Eq. (22) along with the Monte Carlo simulation versus normalized turbulence-free SNR (i.e., $\rho / \gamma_{R}$ ) for various numbers of receive apertures $M=1,2,3,4$ and $J=3$ compensated modes in each aperture. We observe a very good match between analytical and simulation results. ${ }^{4}$ The required SNR to achieve a target outage probability of $10^{-5}$ for $M=1$ is $64 \mathrm{~dB}$ (not shown in the figure for the brevity of presentation). Through the deployment of multiple apertures, we observe impressive performance gains (in terms of power efficiency) of $30,42.5$, and $48 \mathrm{~dB}$, respectively, for $M=2,3$, and 4 in comparison to the benchmark scheme of a single receive aperture system. It is also observed that, in the high-SNR regime, the diversity gain is determined by the number of

${ }^{4}$ Due to the time-consuming nature of Monte Carlo simulations, the simulation results are provided up to $10^{-6}$. 


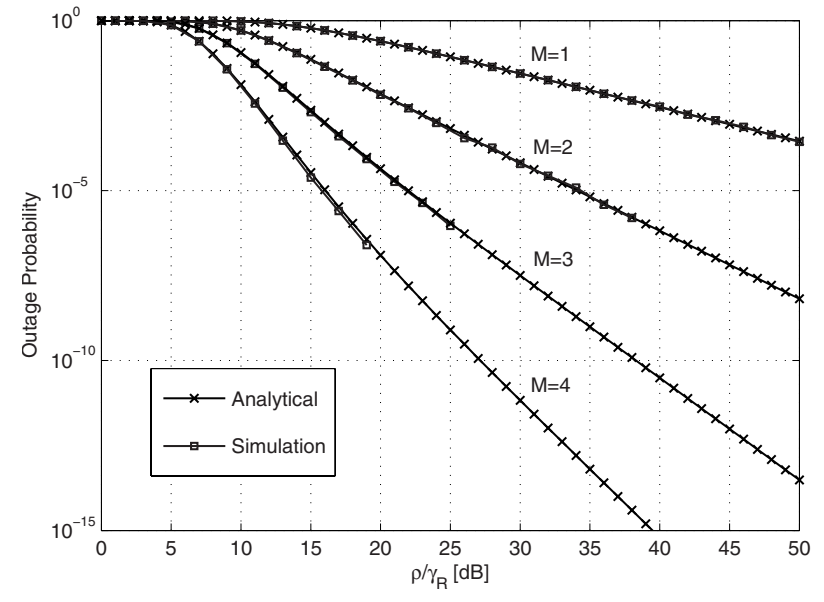

Fig. 3. (Color online) Outage probability for different numbers of receive apertures.

receive apertures as expected from our analytical result given by Eq. (30).

Figure 4 illustrates the finite-SNR DMT given by Eq. (26) for a system with $M=2$ and $J=6$ assuming $\mathrm{SNR}=10,20,30$, $60 \mathrm{~dB}$. It is interesting to note that for practical SNR values, the maximum diversity gain does not occur at zero multiplexing gain. This is mainly due to the presence of the coherent component in the received field, which is a phenomenon also observed in wireless RF systems [27,31]. Recall from Eq. (26) that the finite-SNR DMT is a function of not only the number of receive apertures but also the parameter $K$, which is defined as the ratio of the strength of the coherent component to the incoherent one in the detected field. When the SNR increases, the incoherent (random) component begins to dominate the system performance and the DMT curve gradually approaches its asymptotic value, i.e., the plot labeled with $\mathrm{SNR}=\infty$.

Figure 5 depicts the finite-SNR diversity gain for a fixed data rate given by Eq. (29) assuming a different number of receive apertures $M=1,2,3,4$ and $J=3$. It is observed that, for $M=1$, the maximum diversity gain is achieved in the high-SNR regime. In this case, the strength of the coherent

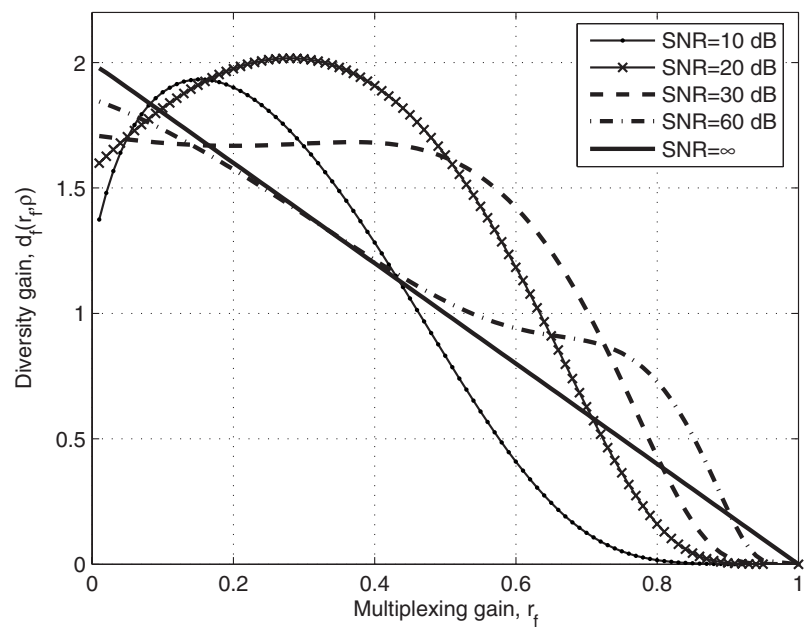

Fig. 4. Finite-SNR DMT for various values of SNR $(M=2)$.

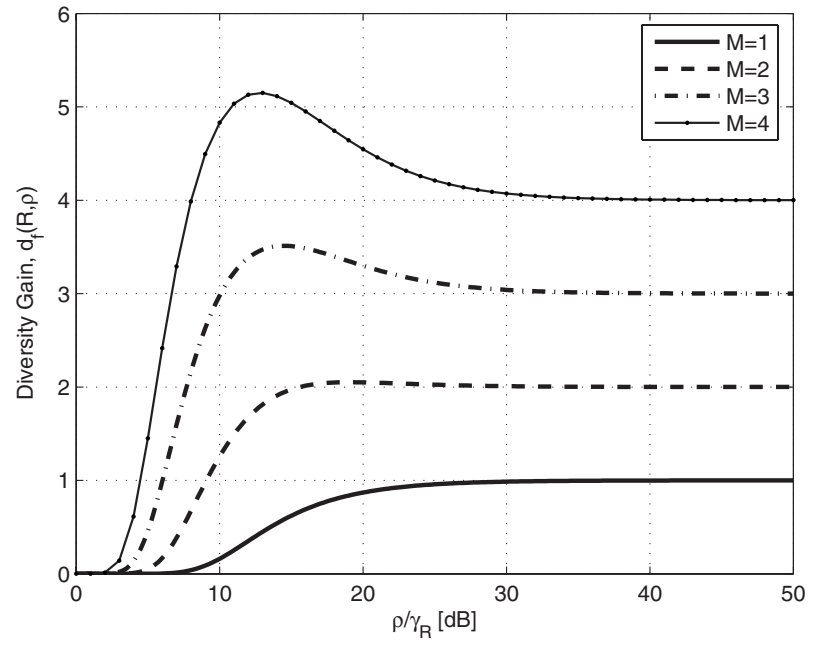

Fig. 5. Diversity gain (at a fixed transmission rate) for various numbers of receive apertures.

component to the incoherent one in the detected field (quantified by $K$ ) is so small that the effective channel distribution approaches Rayleigh distribution. As $M$ increases, $K$ improves and consequently, the maximum diversity gain occurs at a finite value of the SNR. In this range of moderate SNRs, the coherent component is the dominating factor and results in a peak in the finite-SNR diversity gain. As the SNR increases, the incoherent component begins to dominate and decreases the diversity gain, which finally converges to the asymptotical one determined by the number of receive apertures.

In Figs. 4 and 5, we have observed the effect of the coherent component (through dependence on $K$ ) on the performance. One can check from Eq. (12) that $K$ is a function of several channel parameters and the number of compensated modes $(J)$, the latter of which is in fact a system design parameter. In Fig. 6, we study the effect of $J$ on the finite-SNR diversity gain. We assume $J=1,6,11,21$ and consider $M$ $=1$. Similar to our observations in Fig. 5 for $M=1$, when $J$ $=1$ (only the "piston" mode is compensated), $K$ is very small,

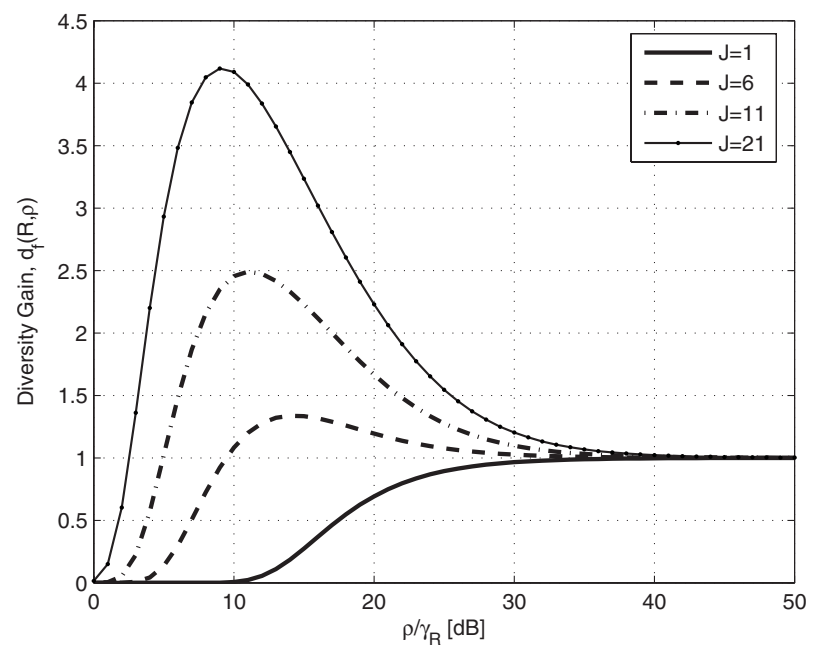

Fig. 6. Effect of the modal compensation on the finite-SNR diversity gain. 
and consequently, the effective channel behaves like Rayleigh distribution and causes maximum diversity gain to occur in the high-SNR regime. But, as $J$ increases, the finiteSNR diversity gain takes larger values. This actually indicates that modal compensation acts as an additional diversity source besides multiple apertures in the finite-SNR regime. Nevertheless, the asymptotic value for all cases converges to the number of receive apertures $(M=1$ in this example) as expected from Eq. (30).

As our results demonstrate, both multiple receivers and modal compensation provide diversity gains. Preference of one method to another or simultaneous deployment depends on the cost and complexity issues. In the absence of atmospheric fading, deployment of a large receive aperture increases the total received signal power and therefore improves the system performance. On the other hand, the presence of atmospheric turbulence causes the incoherency of the received field and this results in significant performance degradation particularly for large apertures. For performance improvement, one can increase the number of compensated modes, but this comes at the cost of higher complexity. Alternatively, one can create an effective large aperture at the receiver by deploying multiple smaller apertures whose total aperture area is equal to that of the large one. When the diameter of each aperture becomes smaller, the received wavefront is more coherent over each aperture compared to the system with a larger aperture. Therefore, only a few first modes can be compensated to yield a desirable performance, and high-order compensation is not required.

\section{CONCLUSION}

In this work, we have analyzed the performance of receive diversity in coherent FSO systems considering both atmospheric turbulence-induced amplitude fluctuation and phase aberration. A modal compensation technique is deployed at each receive aperture to mitigate the turbulence-induced wavefront phase distortion. We have derived closed-form expressions for the diversity gain, which quantifies the link reliability and the DMT, which quantifies the compromise between link reliability and spectral efficiency (i.e., multiplexing gain). For a sufficiently large SNR, we demonstrate that the DMT converges to $M(1-r)$, where $M$ and $r$, respectively, denote the number of receive apertures and multiplexing gain. Asymptotical diversity gain (i.e., DMT evaluated at $r=0$ ) is therefore determined by the number of receive apertures. Our results further demonstrate that for practical SNR values the diversity gain can be larger than $M$ as a result of the phase compensation deployed in the system. In other words, phase compensation acts as an additional diversity source besides the diversity gain offered by the multiple apertures.

\section{REFERENCES}

[1] R. M. Gagliardi and S. Karp, Optical Communications. Wiley, New York, 1995.

[2] D. Kedar and S. Arnon, "Urban optical wireless communication networks: the main challenges and possible solutions," IEEE Commun. Mag., vol. 42, no. 5, pp. S2-S7, May 2004.

[3] V. W. S. Chan, "Free-space optical communication," J. Lightwave Technol., vol. 24, no. 12, pp. 4750-4762, Dec. 2006.
[4] X. Zhu and J. M. Kahn, "Free-space optical communication through atmospheric turbulence channels," IEEE Trans. Commun., vol. 50, no. 8, pp. 1293-1300, Aug. 2002.

[5] S. M. Haas and J. H. Shapiro, "Capacity of wireless optical communications," IEEE J. Sel. Areas Commun., vol. 21, no. 8, pp. 1346-1357, Oct. 2003.

[6] E. Lee and V. Chan, "Part 1: Optical communication over the clear turbulent atmospheric channel using diversity," IEEE J. Sel. Areas Commun., vol. 22, no. 9, pp. 1896-1906, Nov. 2004.

[7] S. G. Wilson, M. Brandt-Pearce, Q. Cao, and J. H. Leveque, "Free-space optical MIMO transmission with Q-ary PPM," IEEE Trans. Commun., vol. 53, no. 8, pp. 1402-1412, Aug. 2005.

[8] I. B. Djordjevic, B. Vasic, and M. A. Neifeld, "Multilevel coding in free-space optical MIMO transmission with q-ary PPM over the atmospheric turbulence channel," IEEE Photon. Technol. Lett., vol. 18, no. 14, pp. 1491-1493, July 2006.

[9] S. M. Navidpour, M. Uysal, and M. Kavehrad, "BER performance of free-space optical transmission with spatial diversity," IEEE Trans. Wireless Commun., vol. 6, no. 8, pp. 28132819, Aug. 2007.

[10] N. Letzepis, I. Holland, and W. Cowley, "The Gaussian free space optical MIMO channel with Q-ary pulse position modulation," IEEE Trans. Wireless Commun., vol. 7, no. 5, pp. 17441753, May 2008.

[11] M. L. B. Riediger, R. Schober, and L. Lampe, "Multiple-symbol detection for photon-counting MIMO free-space optical communications," IEEE Trans. Wireless Commun., vol. 7, no. 12, pp. 5369-5379, Dec. 2008.

[12] T. A. Tsiftsis, H. G. Sandalidis, G. K. Karagiannidis, and M. Uysal, "Optical wireless links with spatial diversity over strong atmospheric turbulence channels," IEEE Trans. Wireless Commun., vol. 8, no. 2, pp. 951-957, Feb. 2009.

[13] W. O. Popoola, Z. Ghassemlooy, J. Allen, E. Leitgeb, and S. Gao, "Free-space optical communication employing subcarrier modulation and spatial diversity in atmospheric turbulence channel," IET Optoelectron., vol. 2, no. 1, pp. 16-23, Feb. 2008.

[14] E. Lee and V. Chan, "Diversity coherent receivers for optical communication over the clear turbulent atmosphere," in IEEE ICC, Glasgow, 2007, pp. 2485-2492.

[15] E. Lee and V. Chan, "Diversity coherent and incoherent receivers for free-space optical communication in the presence and absence of interference," J. Opt. Commun. Netw., vol. 1, no. 5, pp. 463-483, Oct. 2009.

[16] S. M. Haas, J. H. Shapiro, and V. Tarokh, "Space-time codes for wireless optical communications," EURASIP J. Appl. Signal Process., vol. 3, pp. 1-11, Mar. 2002.

[17] A. Belmonte and J. M. Kahn, "Capacity of coherent free-space optical links using diversity-combining techniques," Opt. Express, vol. 17, no. 15, pp. 12601-12611, July 2009.

[18] A. Belmonte and J. M. Kahn, "Performance of synchronous optical receivers using atmospheric compensation techniques," Opt. Express, vol. 16, no. 18, pp. 14151-14162, Sept. 2008.

[19] E. Biglieri, J. Proakis, and S. Shamai, "Fading channels: information theoretic and communications aspects," IEEE Trans. Inf. Theory, vol. 44, no. 6, pp. 2619-2692, Oct. 1998.

[20] L. Zheng and D. N. C. Tse, "Diversity and multiplexing: a fundamental tradeoff in multiple antenna channels," IEEE Trans. Inf. Theory, vol. 49, no. 5, pp. 1073-1096, May 2003.

[21] R. K. Tyson, Principles of Adaptive Optics. Academic, San Diego, 1991.

[22] R. J. Noll, "Zernike polynomials and atmospheric turbulence," J. Opt. Soc. Am., vol. 66, no. 3, pp. 207-211, 1976.

[23] G. R. Osche, Optical Detection Theory for Laser Applications. Wiley, New York, 2002.

[24] M. P. Cagigal and V. F. Canales, "Generalized Fried parameterafter adaptive optics partial wave-front compensation," $J$. Opt. Soc. Am. A, vol. 17, no. 5, pp. 903-910, May 2000. 
[25] F. Bowman, Introduction to Bessel Functions. Dover, New York, 1958.

[26] A. Goldsmith, Wireless Communications. Cambridge U. Press, New York, 2005.

[27] R. Narasimhan, "Finite-SNR diversity multiplexing tradeoff for correlated Rayleigh and Ricean MIMO channels," IEEE Trans. Inf. Theory, vol. 52, no. 9, pp. 3965-3979, Sept. 2006.

[28] J. G. Proakis, Digital Communication, 4th ed. McGraw Hill, 2001.

[29] M. K. Simon and M.-S. Alouini, Digital Communication Over Fading Channels. Wiley, 2000.

[30] H. F. Inman and E. L. Bradley, "The overlapping coefficient as a measure of agreement between probability distributions and point estimation of the overlap of two normal densities," Commun. Stat: Theory Meth., vol. 18, no. 10, pp. 3851-3874, 1989.

[31] W. Y. Shin, S. Y. Chung, and Y. H. Lee, "Diversity-multiplexing tradeoff and outage performance for Rician MIMO channels," IEEE Trans. Inf. Theory, vol. 54, no. 3, pp. 1186-1196, Mar. 2008.

Sahar Molla Aghajanzadeh received the B.Sc. and the M.Sc. degrees in electrical engineering from Sharif University of Technology, Tehran, Iran, in 2003 and 2006, respectively. She is currently a $\mathrm{Ph}$.D. student in the department of Electrical and Computer Engineering of the University of Waterloo, Waterloo, Canada. Her research interests include free-space optical communication, cooperative communication, and fading mitigation techniques in wireless optical channels.

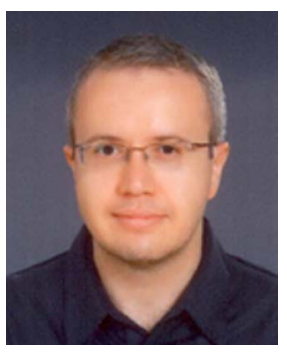

Murat Uysal was born in Istanbul, Turkey in 1973. He received the B.Sc. and the M.Sc. degrees in electronics and communication engineering from Istanbul Technical University, Istanbul, Turkey, in 1995 and 1998, respectively, and the Ph.D. degree in electrical engineering from Texas A\&M University, College Station, Texas, in 2001. Since 2002, he has been with the Department of Electrical and Computer Engineering, University of Waterloo, Canada, where he is now an Associate Professor. He is currently on leave at Özyeğin University, Istanbul, Turkey. His general research interests lie in communications theory and signal processing for communications with special emphasis on wireless applications. Specific research areas include MIMO communication techniques, space-time coding, diversity techniques and coding for fading channels, cooperative communication, and free-space (wireless) optical communication. Dr. Uysal is an Associate Editor for IEEE Transactions on Wireless Communications, IEEE Communications Letters, and the Wireless Communications and Mobile Computing (WCMC) journal. In the past he served as a Guest Co-Editor for the WCMC Special Issue on "MIMO Communications" (October 2004) and the IEEE Journal on Selected Areas in Communications Special Issue on "Optical Wireless Communications" (December 2009). Over the years, he has served on the technical program committee of more than 70 international conferences and workshops in the communications area. He recently co-chaired the IEEE ICC'07 Communication Theory Symposium and the CCECE'08 Communications and Networking Symposium. He is currently serving as the Tutorial Chair of IEEE WCNC'11. Dr. Uysal is a Senior IEEE member. 